\title{
Genotyping-by-sequencing of a melon (Cucumis melo L.) germplasm collection from a secondary center of diversity highlights patterns of genetic variation and genomic features of different gene pools
}

Stefano Pavan ${ }^{1 *}$ (D), Angelo Raffaele Marcotrigiano ${ }^{1}$, Elena Ciani², Rosa Mazzeo ${ }^{1}$, Vito Zonno ${ }^{1}$, Valentino Ruggieri ${ }^{3}$, Concetta Lotti ${ }^{4}$ and Luigi Ricciardi ${ }^{1}$

\begin{abstract}
Background: Melon (Cucumis melo L.) is one of the most important horticultural species, which includes several taxonomic groups. With the advent of next-generation sequencing, single nucleotide polymorphism (SNP) markers are widely used in the study of genetic diversity and genomics.

Results: We report the first successful application of genotyping-by-sequencing (GBS) technology in melon. We detected 25,422 SNPs by the analysis of 72 accessions collected in Apulia, a secondary centre of diversity in Southern Italy. Analyses of genetic structure, principal components, and hierarchical clustering support the identification of three distinct subpopulations. One of them includes accessions known with the folk name of 'carosello', referable to the chate taxonomic group. This is one of the oldest domesticated forms of $C$. melo, once widespread in Europe and now exposed to the risk of genetic erosion. The second subpopulation contains landraces of 'barattiere', a regional vegetable production that was never characterized at the DNA level and we show was erroneously considered another form of chate melon. The third subpopulation includes genotypes of winter melon (C. melo var. inodorus). Genetic analysis within each subpopulation revealed patterns of diversity associated with fruit phenotype and geographical origin. We used SNP data to describe, for each subpopulation, the average linkage disequilibrium (LD) decay, and to highlight genomic regions possibly resulting from directional selection and associated with phenotypic variation.

Conclusions: We used GBS to characterize patterns of genetic diversity and genomic features within C. melo. We provide useful information to preserve endangered gene pools and to guide the use of germplasm in breeding. Finally, our findings lay a foundation for molecular breeding approaches and the identification of genes underlying phenotypic traits.
\end{abstract}

Keywords: Cucumis melo, Genetic diversity, Genomics, Genotyping-by-sequencing, Molecular breeding

\footnotetext{
* Correspondence: stefano.pavan@uniba.it

'Department of Soil, Plant and Food Sciences, University of Bari "Aldo Moro",

Via Amendola 165/A, 70126 Bari, Italy

Full list of author information is available at the end of the article
} 


\section{Background}

Melon (Cucumis melo L., $2 \mathrm{n}=2 \times=24$ ) is one of the most important vegetables worldwide. Current world production of melon is over 31 million tons [1] and is prevalently located in Mediterranean and East Asian countries. The intraspecific classification of $C$. melo has been revised several times. The most recent one [2] includes two subspecies, melo and agrestis, and 15 groups or varietas: acidulous, chinensis, conomon, makuwa and momordica (ssp. agrestis), and adana, ameri, cantalupensis, chandalak, chate, dudaim, flexuosus, inodorus, reticulatus and tibish (ssp. melo). The groups cantalupensis and inodorus have the greatest commercial interest [3].

The Apulia region in Southern Italy is an important secondary centre of diversity for $C$. melo. Besides several landraces of winter melon (C. melo var. inodorus), the chate melon is still grown there as a last relic of a former wider cultivation in Europe, and is known with the folk name of 'carosello' [4, 5]. Its fruits, cylindric in shape and typically covered by long trichomes, are harvested unripen and used raw in replacement of cucumbers. Laghetti et al. [6] assigned another typical Apulian vegetable production, referred to as 'barattiere', to the chate group. However, it is readily distinguishable from 'carosello' by its rounder shape and shorter trichomes. Together, germplasm of 'carosello' and 'barattiere' is estimated to be cultivated over a modest area (about $100 \mathrm{ha}$ ), and thus is seriously exposed to the risk of genetic erosion [7].

The study of genetic diversity is of utmost importance to address conservation programmes contrasting the erosion of cultivated gene pools and to guide the use of germplasm in breeding. With the advent of next-generation sequencing (NGS), single nucleotide polymorphism (SNP) markers are commonly used to describe genetic diversity, as they are present in a large number and merge excellent attributes such as wide genomic distribution, high reproducibility and co-dominant inheritance. Today, the genotyping-by-sequencing (GBS) assay is considered one of the most convenient approach for simultaneous large-scale SNP discovery and genotyping [8] and is widely employed to detect polymorphisms in plant species with sequence information $[9,10]$. There are no reports of the application of GBS in melon, although its genome is publicly available [11].

Here, we used a GBS approach for the genetic characterization of Apulian germplasm of C. melo. Besides local landraces of winter melon, several accessions of 'carosello' and 'barattiere' were included in the analysis, and were shown to form clearly distinct gene pools. In addition, SNP data were used to provide information on linkage disequilibrium decay, to highlight regions putatively subjected to directional selection and to carry out a genome-wide association study (GWAS).

\section{Results}

\section{Library sequencing and SNP calling}

Sequencing of a 72-plex GBS library yielded about 160 million good barcoded reads, corresponding to an average of 2,2 million reads/sample. About $66 \%$ of the reads were successfully mapped onto the melon genome. The TASSEL-GBS pipeline [9] yielded 25,422 polymorphisms, supported by an average depth of 67 . About $50 \%$ of the SNPs fell in intergenic regions, $24 \%$ in introns, $20 \%$ in exons and 6\% in UTR regions. SNPs in the coding regions lead to $44.2 \%$ of synonymous, $52.2 \%$ of missense and $3.6 \%$ of non-sense mutations. The observed transition/ transvertion ratio was 1.71 . All the accessions contained less than $20 \%$ of missing data across the whole dataset of SNP loci.

\section{Genetic structure}

An admixture-based clustering model implemented in the software STRUCTURE [12] was used to infer the genetic structure of a germplasm collection representative of the genetic variation of $C$. melo cultivated in Apulia (Additional file 1). Prior to analysis, biallelic SNPs were filtered with stringent parameters (MAF $>0.05$, call rate $>80 \%$, and proportion of heterozygous $<50 \%$ ), resulting in 8,012 high-quality polymorphisms scattered throughout the $13 \mathrm{C}$. melo pseudochromosomes present in the melon 3.5.1. genome assembly [13] (Additional file 2). Moreover, as the model assumes independence of loci, the SNP dataset was pruned on the basis of estimates of pairwise linkage disequilibrium between adjacent markers. A model with three subpopulations $(K=3)$ (Fig. 1a) was the most suitable for the data, as inferred by the estimation of the $\Delta \mathrm{K}$ parameter [14] (Additional file 3). Remarkably, the distribution of the accessions in the three subpopulations fully matched with the phenotypic classification in inodorus, 'carosello' and 'barattiere' (Fig. 2). Thus, the subpopulations were named I, C and B, respectively (Fig. 1a). About $15 \%$ of the accessions was classified of admixed ancestry, as the highest estimate of membership coefficient was lower than 0.6. Overall estimates of the pairwise fixation index ( $\mathrm{F}_{\mathrm{ST}}$ values) were 0.47 between $\mathrm{B}$ and I (95\% confidence boundaries: $0.46-0.48), 0.35$ between $\mathrm{B}$ and $\mathrm{C}$ (95\% confidence boundaries: $0.34-0.36$ ) and 0.27 between $C$ and I (95\% confidence boundaries: 0.26-0.28).

Genetic structure was also studied within each subpopulation. To this aim, the SNP dataset was filtered separately for groups of accessions, resulting in the detection of 10,725 polymorphisms in C, 7,013 in I and 5,353 in B. A model with $\mathrm{K}=3$ best explained stratification within I (Fig. 1b). Each group included accessions with different fruit rind: yellow, green and speckled (Additional file 4). A model with $K=2$ was the most likely for $C$, however no correlation was found between each of the two groups and specific features of the accessions (data not shown). Finally, 

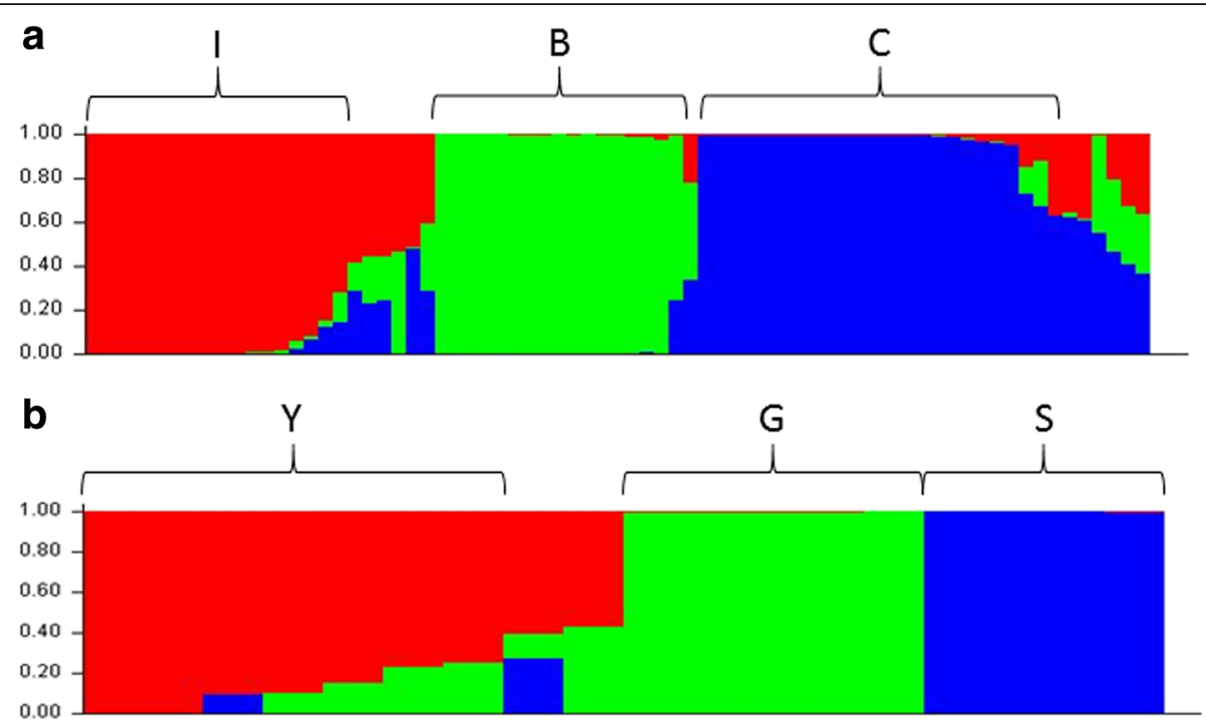

Fig. 1 Population structure analysis. Results are shown for the minimum number of subpopulations $(K)$ which sufficiently define genetic variation, as inferred by the estimation of the $\Delta \mathrm{K}$ parameter. Each individual is represented by a vertical line, which is partitioned into coloured segments whose length depends on the estimated membership fraction (q) in each subpopulation. Individuals are assigned to a specific subpopulation when the highest q is higher than 0.6 (a) Genetic structure of the C. melo germplasm collection used in this study. The subpopulations I, C, and B, which refer to the types inodorus, 'carosello' and 'barattiere', respectively, are indicated. b Genetic structure of the subpopulation I. The three subgroups are named $Y, G$ and $S$ as they contain accessions with yellow, green and speckled fruit rind, respectively

no stratification was detected by the analysis of the subpopulation B.

\section{Genetic relationships among individual accessions}

Principal component analysis (PCA) and NeighbourJoining clustering were performed to identify patterns of genetic variation among individual accessions. Genotypes of I, C and B formed three PCA distinct groups, while admixed accessions were scattered at the center of the PCA plot (Fig. 3a). Further PCAs were performed to understand genetic relationships within each subpopulation. PCA within I revealed the occurrence of three groups, corresponding to accessions with yellow, green and speckled fruit rind (Additional file 5). Interestingly, the upperright panel of the C PCA plot contained all the accessions collected in the province of Lecce, in the southern part of

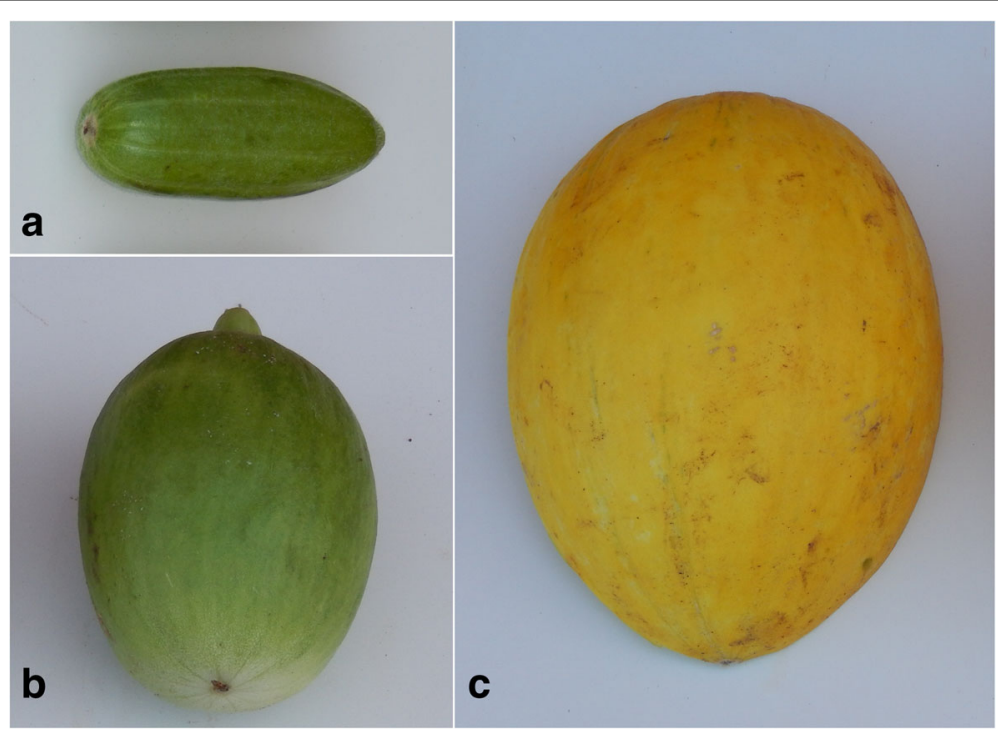

Fig. 2 Typical fruit phenotype of a 'carosello', 'barattiere' (b) and 'inodorus' (c) accessions cultivated in the Apulian center of diversity 


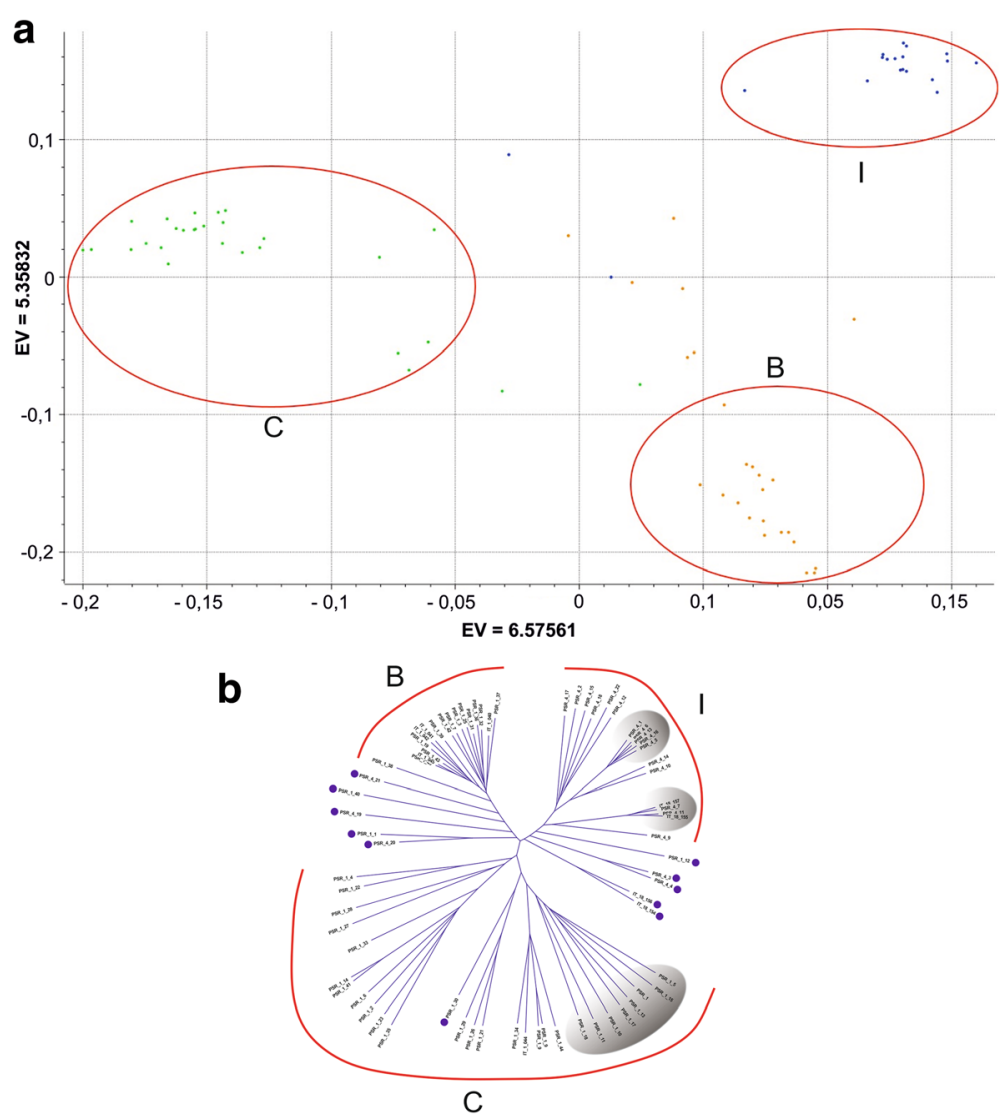

Fig. 3 Genetic relationships within the germplasm collection used in this study. a Principal component analysis (PCA) plot. Different colors represent accessions phenotypically classified as inodorus (orange), 'carosello' (green) and 'barattiere' (blue). Circles delimit groups corresponding to the subpopulations C, B and I identified by structure analysis (b) Neighbour-Joining cladogram. Accessions assigned to the C, B and / subpopulations are highlighted (red lines). Admixed accessions are indicated with dots. Shaded areas indicate the / genetic clusters including accessions with green and speckled fruit rind and the $C$ genetic cluster encompassing all the accessions collected in Southern Apulia

Apulia (Additional file 6). No clear correlation was found between PCA patterns and specific features of B accessions (data not shown).

Neighbour-Joining clustering substantiated the results obtained with structure analysis and PCA. Three main nodes separated the subpopulations I, C and B. Within I, two clusters enclosed accessions with green and speckled rind, respectively; within $C$, a cluster contained all the accessions collected in Southern Apulia (Fig. 3b).

\section{LD decay}

Having shown that the C. melo germplasm collection used in this study is stratified in three distinct subpopulations, we decided to estimate LD decays for each of them separately (Fig. 4). The fastest LD decay was displayed by the subpopulation $\mathrm{C}$, as $\mathrm{r}^{2}$ reached the threshold of 0.2 after $72 \mathrm{~Kb}$. Conversely, the slowest decay was associated with the subpopulation I, in which the same threshold $\mathrm{r}^{2}$ value corresponded to a distance of $774 \mathrm{~kb}$. The subpopulation $\mathrm{B}$ showed an intermediate behavior $\left(r^{2}=0.2\right.$ after $\left.285 \mathrm{~kb}\right)$.

\section{Genomic scan for selection signatures}

We estimated the pairwise fixation index $\left(\mathrm{F}_{\mathrm{ST}}\right)$ parameter at individual SNPs, in order to identify loci putatively subjected to different selection pressures in the subpopulations I, $\mathrm{C}$ and $\mathrm{B} . \mathrm{F}_{\mathrm{ST}}$ approaches the upper limit of 1 when two subpopulations tend to fix opposite alleles. Thirty-three highly divergent loci $\left(\mathrm{F}_{\mathrm{ST}}>0.9\right)$ were identified between the subpopulations $C$ and I, 81 between $C$ and $B$ and 411 between B and I (Fig. 5 and Additional file 7). Two adjacent divergent loci on chromosome 6 (6:1819634-SNV and 6:1819635-SNV), positioned within the putative HOPM interactor 7 homolog MELO3C006224T1, define alleles which are private to the $\mathrm{B}$ group $\left(\mathrm{F}_{\mathrm{ST}}\left[\mathrm{C}_{\mathrm{Cs}} \mathrm{B}\right]=1\right.$ and $\mathrm{F}_{\mathrm{ST}}\left[\right.$ [в $_{\mathrm{B}}$ vs $\left.\mathrm{I}\right]=1$ ). Similarly, the intergenic locus 5:27200654-SNV on chromosome 5 defines an allele private to the $\mathrm{I}$ group $\left(\mathrm{F}_{\mathrm{ST}[\mathrm{B} \text { vs I] }}=1\right.$ and $\left.\mathrm{F}_{\mathrm{ST}[\mathrm{C} \text { vs I] }}=1\right)$ (Additional file 7). Several clusters of at least two consecutive markers displaying $\mathrm{F}_{\mathrm{ST}}>0.9$ were observed for all the pairwise comparisons: 70 between $B$ and I, 16 between $C$ and $B$ and 9 between $C$ and I (Additional file 8). The largest cluster refers to the B vs I comparison and 


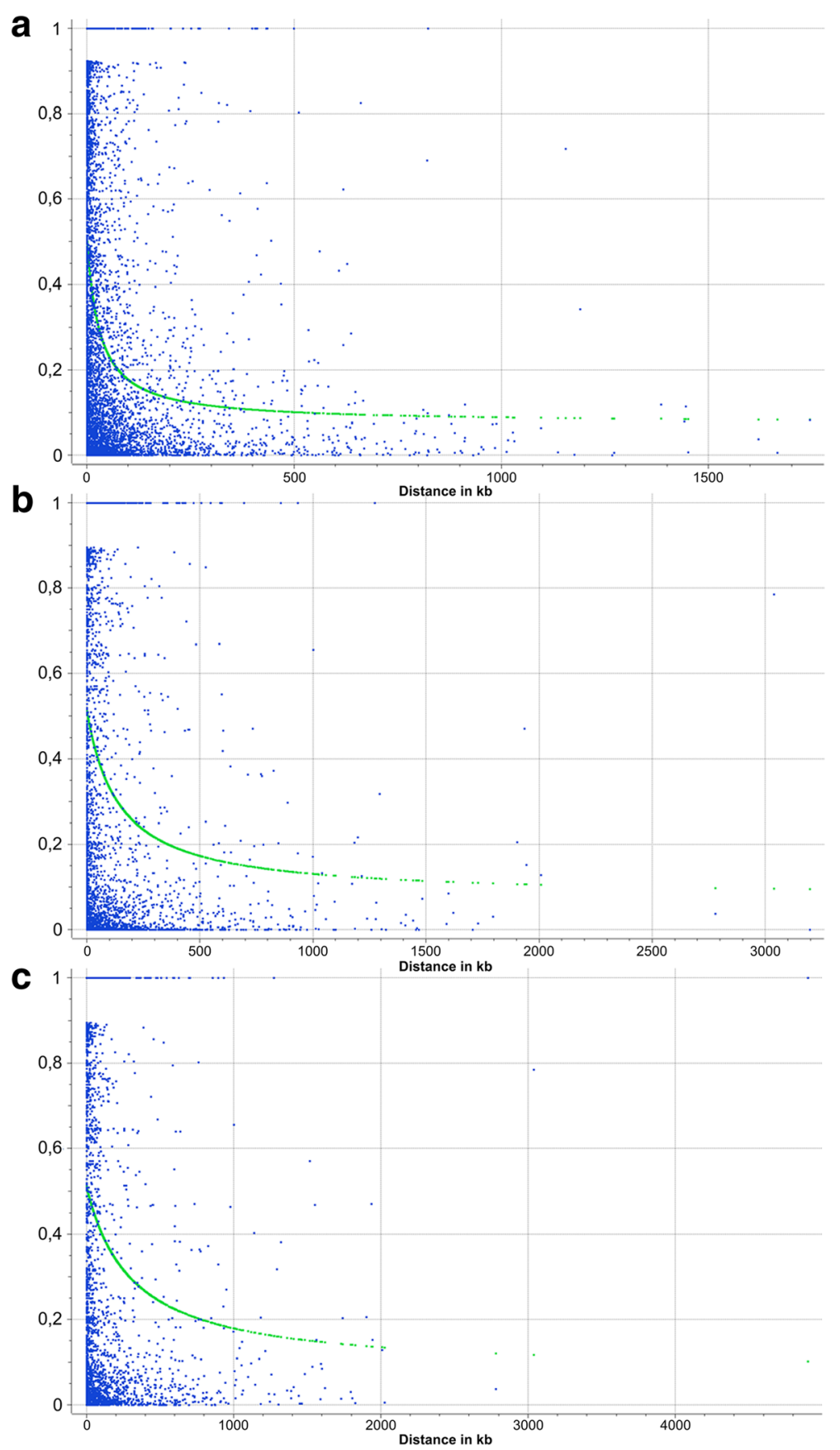

Fig. 4 Average LD decay $\left(r^{2}\right)$ estimated in the C. melo subpopulations $C(\mathbf{a}), B(\mathbf{b})$ and I (c)

includes 31 loci spanning an interval of $41,791 \mathrm{bp}$ on chromosome 1 (Additional file 8).

\section{Genome-wide association study}

A GWAS assay was performed to search for loci associated with the following morpho-agronomic traits: flowering time both for male and perfect flowers (FLTM and FLTP), seed length (SL), seed width (SW) and 100 seed weight (SWe). FLTM was significantly associated with two SNP loci, 2:689400-SNV and 8:24132601-SNV, on chromosome 2 and 8, respectively (Additional file 9). The former is located within the gene MELO3C015310, encoding a putative rubber elongation factor/small rubber particle protein (Ref/SRPP), while the latter is located in an intergenic region. Two significant associations were also found for SW (Additional file 10), referable to the intergenic locus 2:19079933-SNV, positioned on chromosome 2, and the chromosome 6 locus 6:2464455-SNV, located 


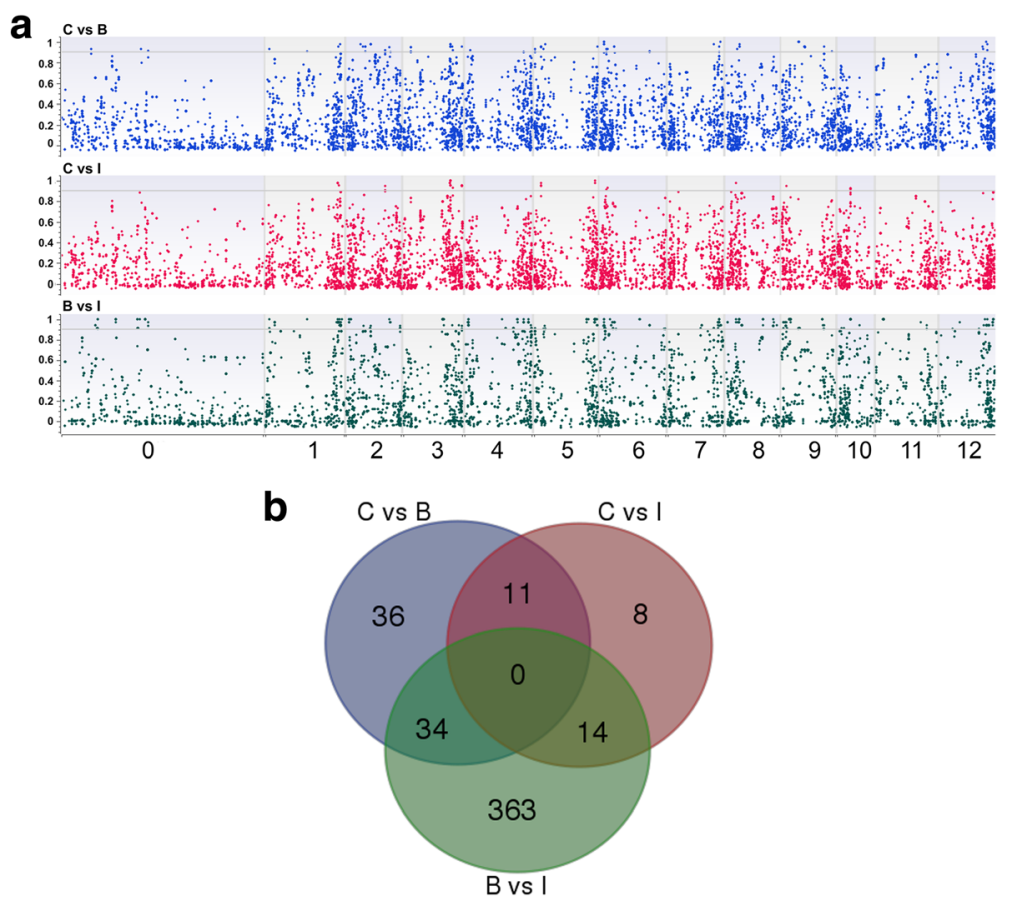

Fig. 5 Analysis of single-loci pairwise $F_{S T}$ estimates among the subpopulations C, B and $I$. a Genomic distribution of 8,012 SNP loci identified in this study in function of pairwise $F_{\text {ST }}$ estimates. The horizontal line on each graph defines highly polymorphic loci associated with $F_{S T}>0.9$ (b) Venn diagram of highly polymorphic loci ( $\left.F_{S T}>0.9\right)$

within the gene MELO3C006314 encoding a putative 60S ribosomal protein L13-a2.

\section{Discussion}

Here we report the first application of GBS, a low-cost and high-throughput approach widely used to explore genetic diversity in cultivated species [15], for the characterization of C. melo germplasm. More than $25 \mathrm{~K}$ polymorphisms were detected, suggesting that GBS could be conveniently used in this species for further characterization of collections and other genomic studies.

Structure analysis indicates that three clearly distinct subpopulations define the genetic variation of the $C$. melo germplasm cultivated in Apulia, a secondary center of diversity in Southern Italy. These include accessions of winter melon and entries of morphotypes known with the folk names of 'carosello' and 'barattiere'. The admixed accessions detected in this study, representing $15 \%$ of the collection, might originate from cross-pollination events during on-farm seed production, ultimately leading to genomic introgressions.

The chate melon has a centuries-old history of cultivation, starting at least from the ancient Egypt [16]. In Italy, it was presumably present already during the Roman Empire, although the first written documents indicating its cultivation date back to the Middle Age $[17,18]$. In contrast with its past wide distribution, today chate melons are found in a few local markets. This study, providing a first molecular characterization of the chate germplasm, might be of great interest to preserve this taxonomic group from genetic erosion. Interestingly, PCA and hierarchical clustering analyses indicate that patterns of genetic diversity of Apulian chate melons also depend on their geographical origin, and this information could be conveniently used to orient germplasm conservation actions and breeding.

Prior to this study, landraces known as 'barattiere', despite being morphologically distinct from those of 'carosello', were also assigned to the chate taxonomic varietas [6]. The results of this study question this notion, as the $\mathrm{B}$ and $\mathrm{C}$ subpopulation form clearly distinct genetic pools. Moreover, the genetic distance between $\mathrm{B}$ and $\mathrm{C}$ (estimated by $\mathrm{F}_{\mathrm{ST}}$ ) is higher than the distance observed between melons belonging to the different varietas chate ('carosello') and inodorus (winter melon). Possibly, the confusion between the two types arose from their common use as unripen fruits. It would be extremely interesting to compare, at the genomic level, 'barattiere' with all the intraspecific varietas reported for C. melo [2]. This might provide insights into the taxonomy of $C$. melo and its cultivation history.

The inodorus group is traditionally cultivated in Southern Italy, where it is appreciated for its long storability that allows consumption during the whole autumn and winter [19]. Here, we show that the distribution of molecular diversity of inodorus accessions is associated with the rind 
phenotype. This finding is consistent with a previous cluster analysis carried out on Spanish and Eurasian germplasm [3]. Yellow and green rind accessions collected in this study are morphologically similar to the Spanish 'Amarillo' and 'Tendral' types, respectively. Close genetic relationships between Italian and Spanish inodorus germplasm, which might be unveiled by future studies, are likely to occur as Southern Italy experienced a long Spanish domination during the Early Modern period.

Another aim of this work was to study the average LD decay in C. melo germplasm, an information of great importance for GWAS. Tomason et al. [20] previously used genome-wide data for the quantification of average LD decay in C. melo. However, these authors pooled several taxonomic groups and used simple sequence repeat (SSR) markers, therefore their results are not comparable to those reported in this study. The subpopulation I displayed a much slower LD decay than $\mathrm{C}$ and $\mathrm{B}$. This might be explained by the reasonable assumption that winter melon experienced a stronger anthropic selection pressure than 'carosello' and 'barattiere', which led to the fixation of a higher number of LD blocks. The slow LD decay in the inodorus group is ideal to identify markers associated with favourable traits, and thus assisted selection, as it allows for an efficient coverage of the genome with a relatively low number of markers. On the other hand, long LD blocks may represent a limiting factor when association studies are aimed to fine-map genes of interest.

An additional goal of this study was to detect putative selection signatures in the genome of the three C. melo subpopulations characterized by structure analysis. Thus, several highly divergent SNP loci were identified by the estimation of pairwise $\mathrm{F}_{\mathrm{ST}}$ values. As we showed that LD decays at a low rate in C. melo, the chance is high that these loci are not the real targets of selection, but are rather in association with them. Unfortunately, knowledge on the molecular basis underlying phenotypic variation in C. melo is still scarce. Moreover, the B and $\mathrm{C}$ groups, which are of local commercial interest, lack a thoroughly phenotypic characterization. Filling these gaps might thus help to associate high $\mathrm{F}_{\mathrm{ST}}$ loci identified in this study with specific events of selection.

Previous works successfully used populations of moderate size for GWAS [21-23]. Therefore, we decided to use our germplasm collection for a medium-resolution association study, whose results might be integrated by further investigations taking into account larger samples and replicated trials. To reduce the amount of falsepositives, we used a weighted mixed linear model (MLM) taking into account kinship and genetic structure $(K+Q)$, which was proven useful in GWAS [24-27]. Moreover, to increase statistical stringency, we considered loci with high minimum allele frequency $(>10 \%)$ and used the Bonferroni correction. Significant associations were detected for seed width and flowering time. These two traits are wellstudied in plants, although little information is so far available in melon $[28,29]$. Interestingly, a putative GID1c gibberellin receptor (MELO3C015362) maps 400Kbp far from the marker 2:689400-SNV, associated with FLTM. In Arabidopsis, GID1 homolog mutants are extremely late flowering under long-day conditions and fails to flower under short-day conditions [30, 31]. In addition, a putative CLEAVAGE STIMULATION FACTOR (MELO3C008872), whose mutation leads to late flowering in Arabidopsis [32], is located $800 \mathrm{Kbp}$ far from the locus 8:24132601-SNV, also associated with FLTM in this study. A candidate gene was also found for SW, as an homolog of the MULTICOPY SUPRESSOR OF IRA1 (MSI1) gene (MELO3C006243), previously associated with seed development [33], resides 490 Kbp far from the SNP locus 6:2464455-SNV. Functional studies targeting candidate genes identified in this study might prove their causal link with phenotypic variation in melon.

\section{Conclusions}

GBS was for the first time applied in C. melo. We provide useful information to understand the genetic structure of this species and to protect minor gene pools from genetic erosion. Finally, our results might prompt molecular breeding approaches and be a resource for future studies aiming to link genomic variation with evolutionary and phenotypic traits.

\section{Methods}

\section{Plant material}

A set of 72 accessions of $C$. melo was obtained by local donors, with their prior informed consent, within the framework of the project "Biodiversity of Apulian vegetable species" (Rural Development Programme, European Agricultural Fund for Rural Development, Reg. EC. No. 1698/ 2005), aimed at the safeguard and characterization of Apulian rural biodiversity. The accessions, corresponding to winter melons (25) and the commercial types 'carosello' (28) and 'barattiere' (19) (Additional file 1), are available at the germplasm bank of the Department of Plant, Soil and Food Science of the University of Bari (Italy) and are managed in accordance with the Italian guidelines for the conservation of agricultural biodiversity (http://www.reterurale.it/flex/cm/pages/ServeBLOB.php/L/IT/IDPagina/ 9580) and the FAO Genebank Standards for Plant Genetic Resources for Food and Agriculture (http://www.fao.org/ 3/a-i3704e.pdf).

\section{GBS assay and SNP filtering}

Genomic DNA was isolated from young leaf samples using the DNeasy Plant Mini Kit (Qiagen). A reduced representation GBS library was prepared using the restriction 
enzyme ApeKI as described by Elshire et al. [8] and sequenced (single-end reads) using Illumina HiSeq 2500. The TASSEL-GBS pipeline [9] and the melon 3.5.1. genome assembly [13] were used to call SNPs from uniquely aligned reads and generate an hapmap file. Besides default parameters, a depth ranging from 10 to 300 and a minimum quality score of 20 were imposed. Functional and structural annotation of variants were performed using SnpEff 4.2. [34]. Additional filters were applied to select a subset of SNPs for subsequent genetic analyses. In more detail, biallelic SNPs were filtered for minor allele frequency (MAF) higher than 5\%, call rate higher than $80 \%$ and proportion of heterozygous lower than $50 \%$, using TASSEL v5.2.20 [35]. The filtering procedure was applied on the whole germplasm collection or on each of the three subpopulations identified by structure analysis, depending on the input dataset required for downstream analyses.

\section{Genetic structure and molecular diversity among individual accessions}

The admixture-based clustering model implemented in the software STRUCTURE 2.3.4 [12] was used to estimate the number of hypothetical subpopulations $(\mathrm{K})$ and the probability of individual accessions to fall in each subpopulation. As the STRUCTURE algorithm assumes independent loci, the SNP dataset was pruned prior to analysis based on pairwise linkage disequilibrium (LD) between adjacent markers. This was estimated using the SNP \& Variation Suite (SVS) software v8.4.0 (Golden Helix Inc.), setting the threshold for $\mathrm{r}^{2}$ equal to 0.5 . Each $\mathrm{K}$ was run ten times with a burn-in period of 100,000 and 100,000 Markov chain Monte Carlo $(\mathrm{MCMC})$ repeats after burn-in. The value of the $\Delta \mathrm{K}$ parameter, based on the second order rate of change of the likelihood function $(\ln \operatorname{Pr}(\mathrm{X} \mid \mathrm{K}))$, was used as criterion to estimate the true K [14]. Genotypes were assigned to one of the subpopulations when the value of the corresponding membership coefficient (q) was higher than 0.6. If not, they were considered admixed.

LD-pruned SNPs were also used to study molecular diversity among individual accessions. Principal component analysis (PCA) was performed using SVS v8.4.0. Furthermore, a Neighbor-Joining cladogram was obtained using the Archaeopteryx visualization tool implemented in TASSEL v5.2.20.

\section{LD decay}

LD decay was evaluated within the three C. melo subpopulations identified by structure analysis. Pairwise $r^{2}$ values, estimated using the expectation-maximization (EM) algorithm implemented in SVS v8.4.0, were plotted against the distance $(\mathrm{kb})$ between adjacent SNP loci, and a regression curve was fit to the data.

\section{$F_{S T}$ analysis}

For each subpopulation pair, fixation index $\left(\mathrm{F}_{\mathrm{ST}}\right)$ estimates were obtained using the formula of Weir and Cockerham [36] available in SVS v8.4.0. Confidence intervals around the $\mathrm{F}_{\mathrm{ST}}$ value were estimated using the percentile-t bootstrapping technique reported by Leviyang and Hamilton [37], implemented by the same software. The average $\mathrm{F}_{\mathrm{ST}}$ provided a measure of genetic distance between subpopulations. $\mathrm{F}_{\mathrm{ST}}$ values at individual loci were plotted against the melon 3.5.1. genome assembly to highlight genomic regions putatively subjected to directional selection. SNP loci associated with $\mathrm{F}_{\mathrm{ST}}$ estimates higher than 0.9 were used to draw a custom Venn diagram.

\section{Association mapping}

Phenotypic traits (FLTM, FLTP, SL, SW, and SWe) were collected on plant material sown at the experimental farm "P. Martucci" of the University of Bari $\left(41^{\circ} 01^{\prime} 22.1^{\prime \prime} \mathrm{N}\right.$ $\left.16^{\circ} 54^{\prime} 21.0^{\prime \prime} \mathrm{E}\right)$, according to a randomized block design with 3 replicates. FLTM and FLTP were recorded as the number of days from the sowing date to the date when $50 \%$ of the plants showed the first flower completely open. Cross-pollination among accessions was prevented using net cages in which bumble bees were introduced. SL, SW and SWe were determined on 100 seeds collected on 10 randomly chosen individuals. Association between SNP loci with a minimum MAF of $10 \%$ and phenotypes was determined using the weighted mixed linear model (MLM) implemented in TASSEL v5, taking into account kinship and genetic structure $(K+Q)$. Significant associations were inferred using an adjusted p-value (Bonferroni correction).

\section{Additional files}

Additional file 1: Accessions of Cucumis melo genotyped in this study. Abbreviation in the origin field refer to different Provinces within the Apulia Region (BA: Bari; BAT: Barletta-Andria-Trani; BR: Brindisi; TA: Taranto; LE: Lecce). (XLSX 78 kb)

Additional file 2: Genomic distribution of the 8,012 high-quality SNPs obtained by GBS analysis of the C. melo germplasm collection used in this study. (PPTX $53 \mathrm{~kb}$ )

Additional file 3: Delta $\mathrm{K}$ distribution from STRUCTURE analysis. $\mathrm{K}=3$ shows a peak indicating that three sub-populations sufficiently define genetic variation in the C. melo germplasm collection considered in this study. (PPTX $48 \mathrm{~kb}$ )

Additional file 4: Yellow (a), green (b) and speckled (c) rind phenotype of C. melo var. inodorus accessions collected in this study. (PPTX 3164 kb)

Additional file 5: Principal component analysis of the I subpopulation. Accessions with yellow, green and speckled rind are represented by yellow, green and blue dots, respectively. (PPTX $41 \mathrm{~kb}$ )

Additional file 6: Principal component analysis of the $C$ subpopulation. Accessions originating from the Southern area of Apulia are highlighted with a circle. (PPTX $49 \mathrm{~kb}$ )

Additional file 7: List of highly divergent loci $\left(F_{S T}>0.9\right)$ in pairwise comparisons among the subpopulations C, B and I. The name of each locus includes the chromosome, the chromosomal position, and the type of polymorphism (SNV = single nucleotide variation; Ins: single nucleotide 
insertion; Del: single nucleotide deletion). For SNP loci falling in putative genes sequences, gene ID and functional annotation reported in the Melonomics database (www.melonomics.net) are indicated. Loci which define alleles private to one of the subpopulations are highlighted in red. (XLSX $67 \mathrm{~kb}$ )

Additional file 8: Clusters of at least two consecutive markers displaying $\mathrm{F}_{\text {ST }}>0.9$ in pairwise comparisons among the subpopulations B, C and I. (XLSX $9 \mathrm{~kb}$ )

Additional file 9: Manhattan plot of the genome-wide association study for flowering time of male flowers (FLTM). Chromosome coordinates are displayed along the $X$-axis. For each locus, the negative log 10 of the $p$-value for association is displayed on the Y-axis. (BMP $1318 \mathrm{~kb}$ )

Additional file 10: Manhattan plot of the genome-wide association study for seed width (SW). Chromosome coordinates are displayed along the $\mathrm{X}$-axis. For each locus, the negative log 10 of the $p$-value for association is displayed on the Y-axis. (BMP $1318 \mathrm{~kb}$ )

\section{Abbreviations}

EM: Expectation-maximization; FLMP: Flowering time of perfect flowers; FLTM: Flowering time of male flowers; GBS: Genotyping by sequencing; GWAS: Genome-wide association studies; LD: Linkage disequilibrium; MAF: Minor allele frequency; MCMC: Markov chain Monte Carlo; MLM: Mixed linear model; NGS: Next generation sequencing; PCA: Principal component analysis; SL: Seed length; SNP: Single nucleotide polymorphism; SSR: Simple sequence repeat; SW: Seed width; SWe: 100 seed weight

\section{Acknowledgements}

Not applicable.

\section{Funding}

This work was funded by the Regional Apulian project Biodiversity of Apulian vegetable species - BiodiverSo" (P.S.R. Puglia 2014-2020), CUP H92C15000270002.

\section{Availability of data and materials}

Raw sequencing FASTAQ files for every sample are available in the NCBI-SRA repository and are accessible through the BioProject number PRJNA327175 (http://www.ncbi.nlm.nih.gov/bioproject/PRJNA327175).

\section{Authors' contributions}

$\mathrm{SP}, \mathrm{CL}$ and $\mathrm{LR}$ designed the experiment. SP, EC, and VR performed the analyses; $\mathrm{LR}$ and $\mathrm{CL}$ established the germplasm collection. ARM, RM and VZ prepared samples for the GBS assay and collected phenotypic data. SP wrote the manuscript. EC, CL and LR critically revised the manuscript. All authors read and approved the final manuscript.

\section{Competing interests}

The authors declare that they have no competing interests.

\section{Consent for publications}

Not applicable.

\section{Ethics approval and consent to participate}

Not applicable.

\section{Author details}

'Department of Soil, Plant and Food Sciences, University of Bari "Aldo Moro", Via Amendola 165/A, 70126 Bari, Italy. ${ }^{2}$ Department of Biosciences, Biotechnologies and Biopharmaceutics, University of Bari "Aldo Moro", Via Amendola 165/A, 70126 Bari, Italy. ${ }^{3}$ Sequentia Biotech, Barcelona, Spain. ${ }^{4}$ Department of the Sciences of Agriculture, Food and Environment, University of Foggia, via Napoli 25, I-71100 Foggia, Italy.

Received: 7 September 2016 Accepted: 16 December 2016 Published online: 09 January 2017

\section{References}

1. FAOSTAT database. Food and Agriculture Organization of the United Nations. http://faostat3.fao.org. Accessed 4 May 2016.

2. Pitrat M. Melon. In: Prohens J, Nuez F, editors. Handbook of plant breeding. Vegetables I. Asteraceae, Brassicaceae, Chenopoidicaceae, and Cucurbitaceae. USA: Springer; 2008. p. 283-315.

3. Esteras C, Formisano G, Roig C, Díaz A, Blanca J, Garcia-Mas J, et al. SNP genotyping in melons: genetic variation, population structure, and linkage disequilibrium. Theor Appl Genet. 2013;126:1285-303.

4. Hammer K, Hanelt P, Perrino P. Carosello and the taxonomy of Cucumis melo L. especially of its vegetable races. Kulturpflanze. 1986;34:249-59.

5. Monforte AJ, Diaz Al, Caño-Delgado A, van der Knaap E. The genetic basis of fruit morphology in horticultural crops: lessons from tomato and melon. J Exp Bot. 2014;65:4625-37.

6. Laghetti G, Accogli R, Hammer K. Different cucumber melon (Cucumis melo L.) races cultivated in Salento (Italy). Genet Resour Crop Ev. 2008;55:619-23.

7. Elia A, Santamaria P. Biodiversity in vegetable crops, a heritage to save: the case of the Puglia Region. Italian J Agron. 2013;8:21-34

8. Elshire RJ, Glaubitz JC, Sun Q, Poland JA, Kawamoto K, Buckler ES, et al. A robust, simple genotyping-by-sequencing (GBS) approach for high diversity species. PLoS One. 2011;6:e19379.

9. Glaubitz JC, Casstevens TM, Fei L, Harriman J, Elshire RJ, Sun Q. TASSEL-GBS: a high capacity genotyping by sequencing analysis pipeline. PLoS One. 2014;9:e90346

10. He J, Zhao X, Laroche A, Lu ZX, Liu H, Li Z. Genotyping-by-sequencing (GBS), an ultimate marker-assisted selection (MAS tool to accelerate plant breeding. Front Plant Sci. 2014;5:484.

11. Garcia-Mas J, Benjak A, Sanseverino W, Bourgeois M, Mir G, González VM, et al. The genome of melon (Cucumis melo L.). Proc Natl Acad Sci U S A. 2012:109:11872-7.

12. Pritchard JK, Stephens M, Donnelly P. Inference of population structure using multilocus genotype data. Genetics. 2000;155:945-59.

13. Argyris JM, Ruiz-Herrera A, Madriz-Masis P, Sanseverino W, Morata J, Pujol M, et al. Use of targeted SNP selection for an improved anchoring of the melon (Cucumis melo L.) scaffold genome assembly. BMC Genomics. 2015;16:4.

14. Evanno G, Regnaut S, Goudet J. Detecting the number of clusters of individuals using the software STRUCTURE: a simulation study. Mol Ecol. 2005:14:2611-20.

15. Deschamps S, Llaca V, May GD. Genotyping-by-sequencing in plants. Biology. 2012;1:460-83.

16. Janick J, Paris HS, Parrish DC. The cucurbits of mediterranean antiquity: identification of taxa from ancient images and descriptions. Ann Bot. 2007:100:1441-57.

17. Paris HS, Janick J, Daunay MC. Medieval herbal iconography and lexicography of Cucumis (cucumber and melon, Cucurbitaceae) in the Occident, 1300-1458. Ann Bot. 2011;108:471-84.

18. Paris HS, Janick J, Daunay MC. Medieval emergence of sweet melons, Cucumis melo (Cucurbitaceae). Ann Bot. 2012;110:23-33.

19. Lotti C, Marcotrigiano AR, De Giovanni C, Resta P, Ricciardi A, Zonno V, et al. Univariate and multivariate analysis performed on bio-agronomical traits of Cucumis melo L. germplasm. Genet Resour Crop Ev. 2008:55:511-22.

20. Tomason Y, Nikkamayala P, Levi A, Reddy U. Map-based molecular diversity, linkage disequilibrium and association mapping of fruit traits in melon. Mol Breed. 2013;31:829-41.

21. Galeano C, Cortes A, Fernandez A, Soler A, Franco-Herrera N, Makunde G, et al. Gene-based single nucleotide polymorphism markers for genetic and association mapping in common bean. BMC Genet. 2012;13:48.

22. Gutierrez L, Cuesta-Marcos A, Castro A, von Zitzewitz J, Schmitt M, Hayes P. Association mapping of malting quality quantitative trait loci in winter barley: positive signals from small germplasm arrays. Plant Genome. 2011:4:256-72.

23. Wang M, Sukumaran S, Barkley N, Chen Z, Chen C, Guo B, et al. Population structure and marker-trait association analysis of the US peanut (Arachis hypogaea L.) mini-core collection. Theor Appl Genet. 2011;123:1307-17.

24. Raggi L, Tissi C, Mazzucato A, Negri V. Molecular polymorphism related to flowering trait variation in a Phaseolus vulgaris L. collection. Plant Sci. 2014;215:180-9

25. Ranc N, Muños S, Xu J, Le Paslier MC, Chauveau A, Bounon R, et al. Genome-wide association mapping in tomato (Solanum lycopersicum) is possible using genome admixture of Solanum lycopersicum var. cerasiforme. G3: Genes Genom Genet. 2012;2:853-64. 
26. Ruggieri V, Francese G, Sacco A, D'Alessandro A, Rigano MM, Parisi M, et al. An association mapping approach to identify favourable alleles for tomato fruit quality breeding. BMC Plant Biol. 2014;14:1.

27. Zhang Z, Ersoz E, Lai CQ, Todhunter RJ, Tiwari HK, Gore MA, et al. Mixed linear model approach adapted for genome-wide association studies. Nat Genet. 2010;42:355-60.

28. Bouché F, Lobet G, Tocquin P, Périlleux C. FLOR-ID: an interactive database of flowering-time gene networks in Arabidopsis thaliana. Nucleic Acids Res. 2015. doi:10.1093/nar/gkv1054.

29. Kesavan M, Song JT, Seo HS. Seed size: a priority trait in cereal crops. Physiol Plantarum. 2013;147:113-20.

30. Griffiths J, Murase K, Rieu I, Zentella R, Zhang ZL, Powers SJ, et al. Genetic characterization and functional analysis of the GID1 gibberellin receptors in Arabidopsis. Plant Cell. 2006;18:3399-414.

31. Ariizumi T, Hauvermale AL, Nelson SK, Hanada A, Yamaguchi S, Steber CM. Lifting DELLA repression of Arabidopsis seed germination by nonproteolytic gibberellin signaling. Plant Physiol. 2013;162:2125-39.

32. Liu F, Marquardt S, Lister C, Swiezewski S, Dean C. Targeted 3' processing of antisense transcripts triggers Arabidopsis FLC chromatin silencing. Science. 2010;327:94-7

33. Köhler C, Hennig L, Bouveret R, Gheyselinck J, Grossniklaus U, Gruissem W. Arabidopsis MSI1 is a component of the MEA/FIE Polycomb group complex and required for seed development. EMBO J. 2003;22:4804-14.

34. Cingolani P, Platts A, Wang LL, Coon M, Nguyen T, Wang L, et al. A program for annotating and predicting the effects of single nucleotide polymorphisms, SnpEff: SNPs in the genome of Drosophila melanogaster strain w1118; iso-2; iso-3. Fly. 2012;6:80-92.

35. Bradbury PJ, Zhang Z, Kroon DE, Casstevens TM, Ramdoss Y, Buckler ES TASSEL: software for association mapping of complex traits in diverse samples. Bioinformatics. 2007;23:2633-5.

36. Weir BS, Cockerham CC. Estimating F-statistics for the analysis of population structure. Evolution. 1984;38:1358-70

37. Leviyang S, Hamilton MB. Properties of Weir and Cockerham's Fst estimators and associated bootstrap confidence intervals. Theor Popul Biol. 2011;79:39-52.

\section{Submit your next manuscript to BioMed Central and we will help you at every step:}

- We accept pre-submission inquiries

- Our selector tool helps you to find the most relevant journal

- We provide round the clock customer support

- Convenient online submission

- Thorough peer review

- Inclusion in PubMed and all major indexing services

- Maximum visibility for your research

Submit your manuscript at www.biomedcentral.com/submit

) Biomed Central 